\title{
Reduced Concanavalin A Capping of Neonatal Polymorphonuclear Leukocytes (PMNS)
}

\author{
GREGORY M. KIMURA, MICHAEL E. MILLER, ${ }^{(11)}$ ROSEMARY D. LEAKE, \\ RUKMANI RAGHUNATHAN, and ANTHONY T. W. CHEUNG \\ Department of Pediatrics, UCLA School of Medicine, Harbor-UCLA Medical Center, Torrance, California, and \\ Department of Biology, Loyola-Marymount University, Los Angeles, California, USA
}

\begin{abstract}
Summary
Neonatal polymorphonuclear leukocytes (PMNs) have previously been shown to be chemotactically deficient. To probe the mechanism(s) responsible for this deficiency, we have investigated the phenomenon of concanavalin A-induced capping in neonatal PMNs. PMNs from cord blood of 17 healthy, full-term infants and 17 normal adult volunteers were isolated by standard Ficoll-Hypaque and dextran sedimentation. After incubation with and without colchicine, the cells were reincubated with fluorescein isothiocyanate-Con A, fixed, and prepared in wet mounts. Using a fluorescence microscope, PMNs were identified, and the percentage of the capped cells was counted.

Upon treatment with colchicine, adult PMNs showed a significant increase in the percentage of capped cells. By contrast, the cord blood PMNs showed no significant increase in capping after colchicine treatment. The difference between percentage of PMNs showing colchicine-induced capping in adult and cord blood was highly significant $(P<0.01$; Student's $t$ test).
\end{abstract}

\section{Speculation}

Increasing evidence suggests that a primary developmental deficiency of neonatal polymorphonuclear leukocyte movement plays an important role in the compromised inflammatory response characteristic of this period of life. The data presented in this report strengthen the argument that the level at which the developmental deficiency operates is at the cell membrane. Future studies should be directed towards determining whether this represents a primary membrane abnormality or, rather, reflects a defect of cytoskeletal or other submembranous components. Such knowledge would hopefully lead to improved therapeutic enhancement of the efficiency of neonatal polymorphonuclear leukocyte movement.

Chemotaxis of human polymorphonuclear leukocytes (PMNs) involves interactions between the cell membrane and several cytoplasmic cytoskeletal elements-the microtubules and microfilaments. The precise nature of these relationships, however, is unclear. Among the available in vitro probes for characterizing these interactions is "capping," or the accumulation of ligandreceptor complexes at a localized site (9).

In lymphocytes, capping can be induced by incubation with polyvalent ligands such as antibodies.

Capping in normal human PMNs can also be demonstrated after exposure to the lectin, concanavalin A (Con A). In normal PMNs, however, Con A capping is significantly increased by incubation of cells with an agent such as colchine, a microtubule disrupter (6).

By contrast, PMNs from patients with Chediak-Higashi syndrome do not require preincubation with colchicine and will significantly cap just upon Con A treatment $(6,7)$. Such PMNs also show defective chemotaxis, thereby suggesting an association between capping and movement in PMNs.

PMNs from normal human neonates also show deficient chemotaxis $(2,4,8)$. To better characterize the nature of this defect, we studied capping in neonatal PMNs, both spontaneously and in response to incubation with colchicine.

\section{MATERIALS AND METHODS}

Cord blood from normal human term neonates was obtained immediately after delivery. Venous blood was obtained from normal adult donors. PMNs from either source were isolated by standard Ficoll-Hypaque differential centrifugation and dextran sedimentation (1). The final PMN suspension, which was approximately $90 \%$ pure, was then standardized to a concentration of 1 $\times 10^{6} \mathrm{PMNs}$ per $\mathrm{ml}$ Dulbecco's phosphate buffered saline (PBS). Capping was studied essentially as described by Oliver et al. (6, 7). Aliquots of each PMN sample were incubated either with or without $5 \times 10^{-6} \mathrm{M}$ colchicine (Sigma Chemical Company, St. Louis, MO; lot $28 \mathrm{C}-0030$ ) for $30 \mathrm{~min}$ at $37^{\circ} \mathrm{C}$. Each aliquot was then incubated with $1.25 \times 10^{-5} \mathrm{~g} / \mathrm{ml}$ of fluorescein isothiocyanate conjugated (FITC) Con A (Cappal Laboratories, Inc., Cochranville, PA; lot 11608) for $7 \mathrm{~min}$ and fixed at room temperature with $2 \%$ paraformaldehyde. Before use, the FITC-Con A was purified by affinity chromatography on Sephadex G-50. Excess FITC-Con A was washed from the final preparations with PBS. The PMNs were then resuspended in 10 to $20 \mu \mathrm{l}$ of PBS from which wet mounts were prepared. The pattern of fluorescence was then read utilizing a Zeiss epiiluminator with an FITC interference filter, 450 dichroic mirror, 53 barrier filter, and a 100 neofluar phase objective.

Cells were scored as either capped or uncapped. The capped category included PMNs in which the FITC-Con A had clearly aggregated into one region or pole of the cell (Fig. 1a) or those in which the FITC-Con-A had aggregated into one region and then formed a knob-like projection. The uncapped category included cells in which little localized fluorescence was observed (Fig. 1b) or those in which only random patches occurred (Fig. 1c).

\section{RESULTS}

Seventeen samples each of cord and adult blood were tested. One hundred consecutive PMNs from each sample were scored as capped or uncapped. Slides were coded and read by observers unaware of the code, thereby limiting subjectivity.

The data are shown in Figure 2. As expected, adult PMNs not incubated with colchicine showed only $16 \%$ capped cells. Upon preincubation with colchicine, however, a significant increase to $48 \%$ capped cells was observed. In contrast to the adult PMNs, however, the colchicine-treated cord cells failed to show a significant increase in capping over spontaneous levels. The mean value of spontaneous capping in the cord PMNs was 18 and 22\% when pretreated with colchicine. 

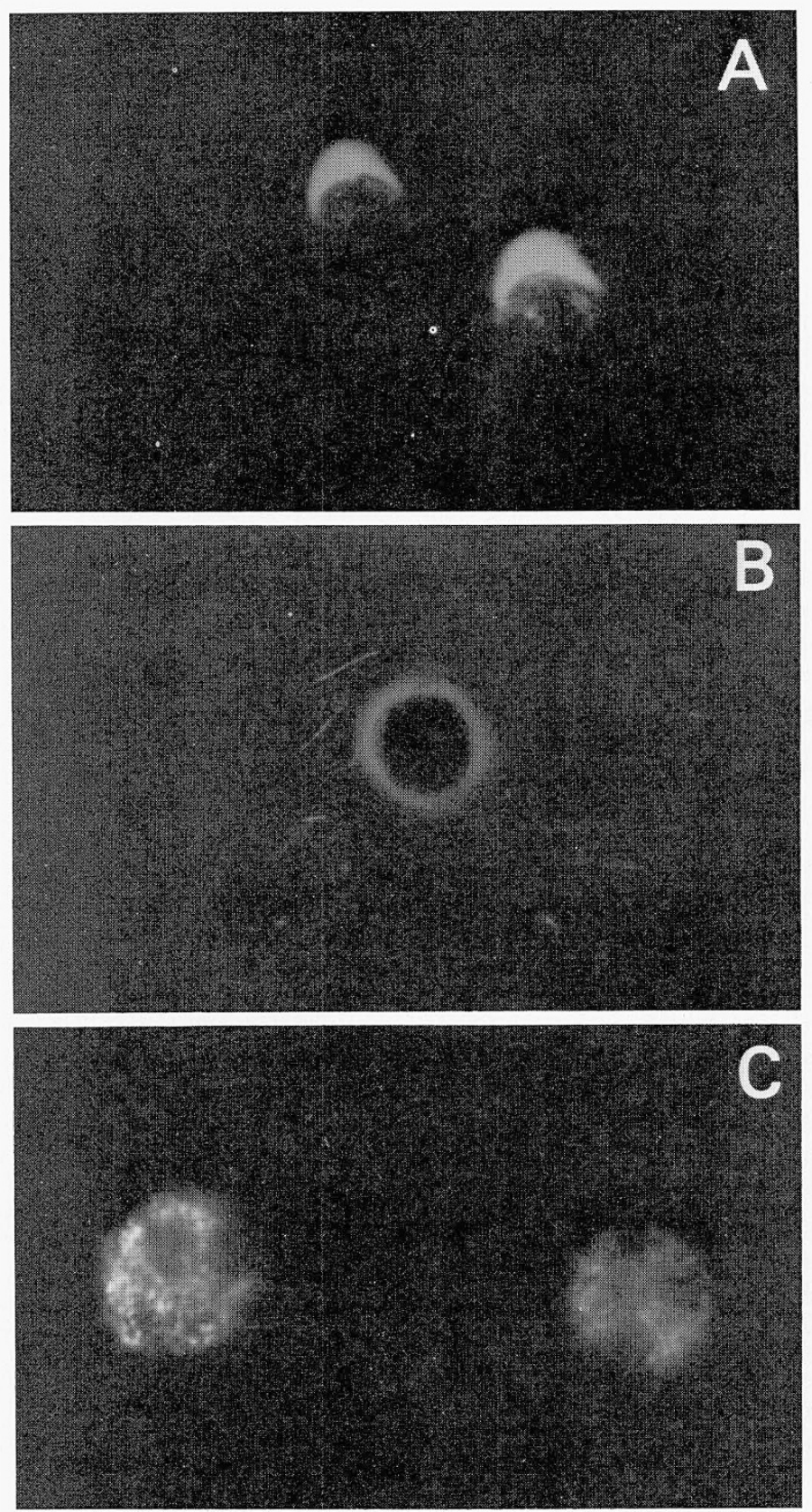

Fig. 1. Patterns of fluorescence of FITC-Con A. $a$, capping; $b$, no localization; and $c$, random patches. See text for details.

To further demonstrate the specificity of the reaction, we treated the cells with $50 \mathrm{mM} \alpha$-methyl mannoside, a competing hapten sugar $(6,7)$. This removed labeling of the diffuse or capped cells, but had no effect upon the patched cells. The latter presumably reflects endocytosed Con $A$, which is inaccessible to the sugar $(6$, 7).

\section{DISCUSSION}

These studies were carried out to further characterize the nature of the movement defect of neonatal PMNs. Previous observations have implicated abnormal membrane activity in neonatal PMNs. In addition to deficient chemotaxis $(2,4,8)$, we have observed decreased deformability of neonatal PMNs (5). Mease et al. (3) have demonstrated decreased phytohemagglutinin-induced aggregation of human PMNs. Together, the findings suggest a developmental membrane or membrane-associated abnormality in the neonatal PMN.
The data in this report lend further support to the concept. Capping reflects membrane fluidity, which in turn reflects submembranous cellular events including the cytoskeletal components of the PMN.

To establish that the observed abnormal capping reflects a true state of the neonatal PMN and not an artifact of either the trauma of childbirth or the uniqueness of cord blood PMNs, several other studies were performed. First, we tested PMN capping in cells from six normal older neonates, ranging in age from 1 day to 4 months. These results were identical to our results from cord blood PMNs. In other words, no significant increase of capping occurred in the older neonatal PMNs treated with colchicine. In another study, we considered whether maternally derived factors which might inhibit cap formation in cord PMNs were present in the blood around the time of delivery. Maternal PMNs were tested just before and after delivery. These results were identical to those of normal adults. The mothers' PMNs demonstrated low sponta- 


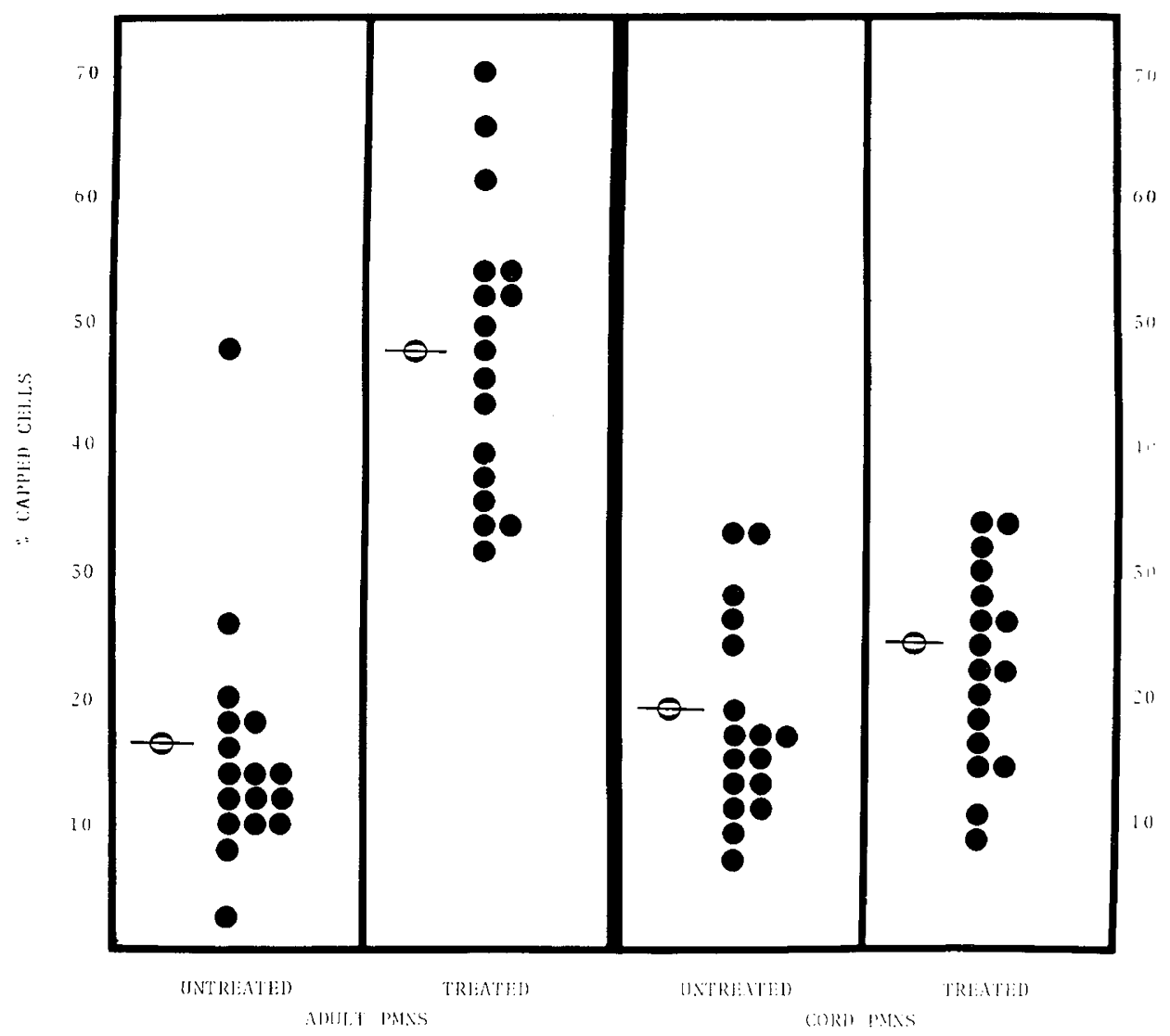

Fig. 2. Effects of colchicine treatment upon Con A capping of adult (left frames) and cord blood (right frames) PMNs. Untreated adult and cord PMNs show comparable degrees of capping. Upon colchicine treatment, adult PMNs show significant increase in capping $(P<0.01 ;$ Student's $t$ test), but cord cells are unaffected.

neous capping with a significant increase upon colchicine treatment.

Finally, the possibility of an inhibitor in cord blood which might impair colchicine-induced capping was further explored by incubating adult cells in cord serum before the study. Such PMNs showed a normal increase in capping after incubation with colchicine, even in the presence of cord serum. This, plus the fact that the first group of studies were carried out utilizing washed, serum-free PMN suspensions provided strong evidence that the defect of colchicine-induced capping is intrinsic to cord blood and neonatal PMNs and not due to a humoral inhibitor.

Thus, decreased capping can now be added to the recognized functional perturbations of the neonatal PMN. Combined with decreased movement $(2,4,8)$, decreased deformability (5), and decreased lectin-induced aggregation of these cells (3), the data strongly point to a developmental functional PMN membrane abnormality. Whether this functional deficiency derives from a primary membrane defect or an indirect effect of submembranous elements upon the membrane remains to be determined.

\section{REFERENCES AND NOTES}

1. Böyum, A.: Isolation of mononuclear cells and granulocytes from human blood Isolation of mononuclear cells by one centrifugation and of granulocytes by combining centrifugation and sedimentation at $1 \mathrm{~g}$. Scand. J. Clin. Lab. Invest. Suppl, 97: 77 (1968).

2. Klein, R. B., Fischer, T. J., Gard, S. E., Biberstein, M., Rich, K. C., and Stiehm,
E. R.: Decreased mononuclear and polymorphonuclear chemotaxis in human newborns, infants, and young children. Pediatrics, 60: 467 (1977).

3. Mease, A. D., Fischer, G, W. Hunter, K. W., and Ruymann, F. B.: Decreased phytohemagglutinin-induced aggregation and C5a-induced chemotaxis of human newborn neutrophils. Pediatr. Res., 14: 142 (1980).

4. Miller, M. E.: Chemotactic function in the human neonate: Humoral and cellular aspects. Pediatr. Res., 5: 487 (1971).

5. Miller, M. E.: Developmental maturation of human neutrophil motility and its realtionship to membrane deformability. In: J. A. Bellanti, D. H. Dayton: The Phagocytic Cell in Host Resistance pp. 295-307 (Raven Press, New York, 1975).

6. Oliver, J. M., and Zurier, R. B.: Correction of characteristic abnormalities of microtubule function and granule morphology in Chediak-Higashi syndrome with cholinergic agonists. J. Clin. Invest., 57: 1239 (1976).

7. Oliver, J. M., Zurier, R. B., and Berlin, R. D.: Con A cap formation on PMN of normal and beige (Chediak-Higashi) mice. Nature (Lond.), 253: 471 (1975).

8. Pahwa, S. G., Pahwa, R., Grimes, E., and Smithwick, E.: Cellular and humoral components of monocyte and neutrophil chemotaxis in cord blood. Pediatr. Res., 11: 677 (1977).

9. Schreiner, G. F., and Unanue, E. R.: Membrane and cytoplasmic changes in B lymphocytes induced by ligand-surface immunoglobulin interaction. Adv. Immunol. 24: 37 (1976).

10. Patient samples: all samples were obtained in accordance and the specific approval of the Human Subjects Committee of Harbor-UCLA Medical Center.

11. Requests for reprints should be addressed to: Michael E. Miller, M.D., Department of Pediatrics, UCLA School of Medicine, Harbor-UCLA Medical Center, 1000 West Carson Street, E-6 Annex, Torrance, CA 90509 (USA).

12. This research was supported by National Institutes of Health, National Institute of Child Health and Human Development, Program Project 1-PO1-HD1097501 Al.

13. Received for publication January 9, 1981

14. Accepted for publication March 17, 1981. 